\title{
Effect of droplet size and counterions on the spatial distribution of ions
}

\author{
Victor Kwan and Styliani Consta* \\ Department of Chemistry, The University of Western Ontario, London, Ontario, Canada \\ N6A $5 B 7$ \\ E-mail: sconstas@uwo.ca
}

\section{Abstract}

Electrosprayed droplets have emerged as a new environment for accelerating reactions by orders of magnitude relative to their bulk analogues. Nevertheless the reaction mechanisms are still unknown. Unraveling of the ion spatial distribution is critical as to where charge transfer reactions are likely to take place and as to their effect on the ionic atmosphere of macroions. Here we investigate the ion spatial distributions in aqueous droplets with diameters in the range of $5 \mathrm{~nm}$ (which is $\approx 1000 \mathrm{H}_{2} \mathrm{O}$ molecules) to $16 \mathrm{~nm}$ (which is $\approx 6 \times 10^{5} \mathrm{H}_{2} \mathrm{O}$ molecules) with and without counterions using molecular dynamics. The charge carriers are $\mathrm{Na}^{+}, \mathrm{Cl}^{-}$ions and model hydronium ions. We scale the distance coordinate of the ion distribution by the Rayleigh radius. The scaled distributions reveal that the smaller the droplet the larger the charge-induced shape fluctuations and the more uniform the ion distribution is toward the droplet center of mass. The model hydronium ions reside in the very outer layers of water, which is strikingly different from the location of $\mathrm{Na}^{+}$and $\mathrm{Cl}^{-}$ions. In the presence of counterions the concentration of the ion in excess increases in the outer water layers of the droplet, relative to that in a droplet with only one type of ion present and the counterions (species in deficiency) are found mainly in the droplet interior. For the first time droplet sizes that are accessible to experimental scrutiny are atomistically modeled.

\section{Introduction}

In the last one and a half decades there has been a surge in the use of microdrops in many chemistry and industry applications, which include dispersive liquid-liquid micro-extraction, ${ }^{1}$ micro-fluidity and acceleration of reactions in electrosprayed droplets. ${ }^{2-8}$ Progressive miniaturization of chemistry has led to the usage of even smaller droplets with a notable example that of nanofluidity. Even though our ability to carry out chemical reactions on mass-scale in these minute volumes is rapidly growing, the knowledge of the reaction mechanisms of the dissolved species in the droplet environment is still limited. The micro-drops and nano-drops are macroscopic and mesoscopic clusters, respectively. ${ }^{7,8}$

Here, we examine the spatial distribution of ions in highly charged nanoscopic droplets found in aerosol phase. This study is significant for several experiments where the droplet chemistry in aerosols plays a central role. These experiments include use of electrosprayed droplets as reaction vessels ${ }^{2-6}$ and droplet-based ionization techniques used in native mass spectrometry. ${ }^{9}$ The spatial distribution of ions is also important in droplets found in atmospheric aerosols. Droplets and particulates in atmospheric aerosols are also charged but not as highly as electrosprayed droplets. ${ }^{10}$ Determining the spatial distribution of the ions is the first step for establishing the ion-evaporation mechanism, ${ }^{11-13}$ which is a principal disintegra- 
tion mechanism of charged droplets in atmospheric and electrosprayed aerosols. The effect of the droplet in the charge state of a macroion is one of the most debated questions in native mass spectrometry. The spatial variation of the ion concentration in droplets may indicate as to where charge transfer reactions that determine the charge state of proteins and nucleic acids may take place in droplets.

Regardless of their charge, droplets in general combine certain characteristics due to their finite-size. These characteristics are: confinement, existence of a large interface with the surrounding medium, large density gradients due to the interface, break-down of translational invariance and shape fluctuations. Depending on the droplet size certain characteristics are more pronounced in certain sizes than others. Notably, shape fluctuations are more pronounced in nano-drops (mesoscopic clusters) than in clusters of a handful of molecules and in macroscopic clusters. The shapes fluctuations are important to consider because they may couple with the reaction mechanisms. ${ }^{7,8}$ Highly charged droplets have additional features to those of neutral droplets. These features are: distinct ion spatial distribution, and thus variable ionic strength throughout the droplet volume, fluctuations pertinent to the charged droplets and charge-induced instabilities when the charge exceeds a threshold value for a certain system size. ${ }^{14-18}$ All of the aformationed characteristics with the possible exception of the charge-induced instability are also present in droplets of atmospheric aerosols. In previous works we have found that the behavior of the ion spatial distribution is captured by a general analytical theory that takes into account a fluctuating droplet interface, an effective screening length of the charges and the finite size of a solvated ion. ${ }^{19}$

In a highly charged droplet we should define the condition of stability. The stability of a charged conducting droplet ${ }^{14-18}$ is determined by the fissility parameter $(X)$ defined as

$$
X=\frac{Q^{2}}{64 \pi^{2} \gamma \varepsilon_{0} R^{3}} .
$$

where $Q$ is the droplet charge, $\gamma$ the surface tension, $\varepsilon_{0}$ and $R$ are the permittivity of vacuum and the radius of the droplet, respectively. When $X$ is less than unity, the system is stable. At $X=1$ the droplet is at the Rayleigh limit, ${ }^{14-18}$ and when $X>1$ the system is unstable. The majority of the droplets that we study are found at $X>0.9$ besides droplets of 880 water molecules that are found at $X \approx 0.57$.

A lot of experience has been obtained over a few decades in modeling ions near a planar interface. ${ }^{20-24}$ These systems are overall neutral, thus we cannot expect a direct transfer of these findings to the case of a charged curved interface. A major force that determines the location of the ions in the droplets is their inherent conducting character.

In this article, we present a systematic study of $\mathrm{Na}^{+}$spatial distributions in aqueous droplets with diameter in the range of $5 \mathrm{~nm}$ (which corresponds to $\approx 1000 \mathrm{H}_{2} \mathrm{O}$ molecules) to $16 \mathrm{~nm}$ (which corresponds to $\approx 6 \times 10^{5} \mathrm{H}_{2} \mathrm{O}$ molecules). For comparison with the $\mathrm{Na}^{+}$ions, the spatial distributions of a model hydronium ion in a droplet of $\approx 1000 \mathrm{H}_{2} \mathrm{O}$ molecules and $\mathrm{Cl}^{-}$and $\mathrm{I}^{-}$in droplets of $\approx 3500 \mathrm{H}_{2} \mathrm{O}$ molecules are presented. We use the $\mathrm{Na}^{+}$spatial distribution as an example to examine the droplet size effect. In the study we attempt to learn whether information on the distributions of ions in smaller droplets can be generalized for larger droplet sizes, which are still not accessible to atomistic simulations.

The new aspects that we examine in this article relative to our previous work ${ }^{19}$ are: (a) the systematic structure of aqueous droplets with positive ions up to a diameter of $16 \mathrm{~nm}$ $\left(\approx 6 \times 10^{4} \mathrm{H}_{2} \mathrm{O}\right.$ molecules $)$. To our knowledge droplets of this size are the largest that have been atomistically modeled thus far and they approach in size those that may be directly emitted by an electrospray source. (b) the location of the negative ions $\left(\mathrm{Cl}^{-}\right.$and $\left.\mathrm{I}^{-}\right)$and their mixture (c) the distinct features of the distribution of model hydronium ions and (d) the effect of counterions.

The presence of the counterions change the ion distribution considerably relative to that of the one type of ions. The concentration of the 
ion in excess increases in the outer water layers of the droplet, relative to the droplet analogue with only the ion in excess present. The counterions (species in deficiency) are found mainly in the droplet interior while they reside mainly in the outer water layers when they are the only charged species present. The model hydronium ions reside in the very outer layers of water, which is strikingly different from the location of $\mathrm{Na}^{+}$and $\mathrm{Cl}^{-}$ions. The location of the hydroniums affects the orientation of the $\mathrm{H}_{2} \mathrm{O}$ molecules in the droplet differently from that of the $\mathrm{Na}^{+}$ions. Droplets with excess $\mathrm{Cl}^{-}$ions show similar structure to that of the $\mathrm{Na}^{+}$ions but the orientation of the water dipoles inverts.

\section{Systems and Simulation Methods}

We performed equilibrium molecular dynamics (MD) simulations of charged aqueous droplets with $\mathrm{Na}^{+}$, a model hydronium ion, $\mathrm{Cl}^{-}$and $\mathrm{I}^{-}$ions. A typical snapshot of a system comprised $\mathrm{H}_{2} \mathrm{O}$ molecules, $\mathrm{Cl}^{-}$and $\mathrm{I}^{-}$ions is shown in Fig. 1. The simulations were performed by using the software NAMD version 2.12. ${ }^{25}$ The Newton's equation of motion for each atomic site was integrated using the velocity-Verlet algorithm with a time step of 1.0 fs. The trajectories were analyzed using VMD 1.9.2. ${ }^{26}$ The water molecules were modeled with the TIP3P (transferable intermolecular potential with 3 points) ${ }^{27}$-CHARMM and the TIP4P $/ 2005^{28}$ and the ions with the CHARMM $36 \mathrm{~m}^{29,30}$ and OPLS (Optimized Potentials for Liquid Simulations), ${ }^{31,32}$ respectively. The TIP3PCHARMM is a m(odified)TIP3P, which is the original TIP3P with Lennard-Jones potential on the hydrogen sites. Hereafter we will use the notation TIP3P for this water model. The parameters of the force fields are presented in Table S1 in the SI.

All the forces were computed directly without any cut-offs. Equilibrium simulations in NAMD were set by placing the droplet in a spherical cavity of radius $20.0 \mathrm{~nm}$ by using spherical boundary condition. The cavity was sufficiently large to accommodate the shape fluctuation of the droplet. The droplet will eventually reach vapor pressure equilibrium. The systems were thermalized with Langevin thermostat with the damping coefficient set to $1 /$ ps. The Rayleigh limit of the droplet (Eq. 1) was calculated with the surface tension values of the water model used at the simulation temperature. ${ }^{33}$ Specifically, for TIP3P at $T=300 \mathrm{~K}$ the value of surface tension is taken to be $0.0523 \mathrm{~N} / \mathrm{m}$ and at $T=350 \mathrm{~K}$ to be $0.0432 \mathrm{~N} / \mathrm{m}$. For TIP4P $/ 2005$ at $T=350 \mathrm{~K}$ is take to be $0.0619 \mathrm{~N} / \mathrm{m}$.

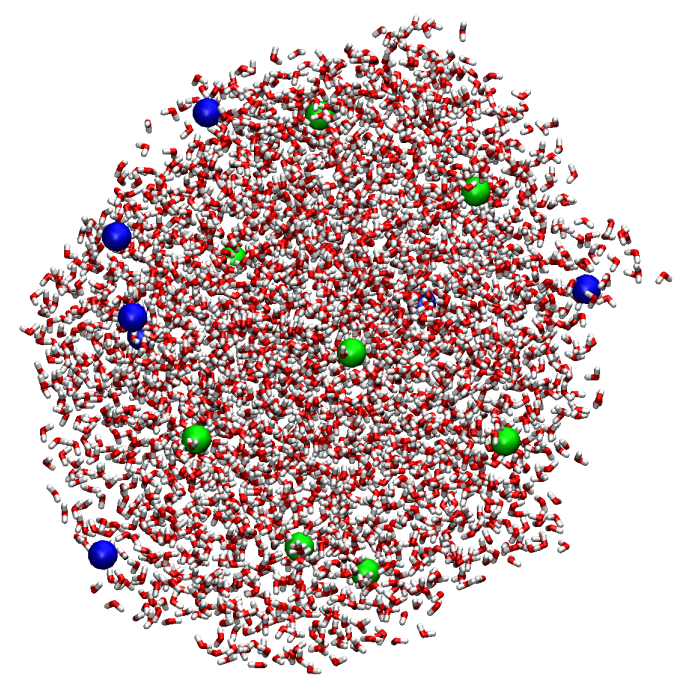

Figure 1: Typical snapshot of a droplet that comprises $\approx 3500$ TIP3P molecules and $8 \mathrm{Cl}^{-}$ ions (green spheres) and $8 \mathrm{I}^{-}$ions (blue spheres).

The system that were examined are shown in Table 1 . The production runs of the $2 \times 10^{4}$ and $3 \times 10^{4} \mathrm{H}_{2} \mathrm{O}$-molecule drops were for $40 \mathrm{~ns}$, following a $10 \mathrm{~ns}$ equilibration time and that of 1000 and $3500 \mathrm{H}_{2} \mathrm{O}$-molecule clusters for $150 \mathrm{~ns}$ and 50 ns, respectively.

A question that may arise concerns the effect of the force field on the ion distributions. The details of the force field lead to different values of macroscopic properties of the solvent (e.g. surface tension, dielecric constant), that enter the general macroscopic models relevant to iondroplet interactions. First of all the surface tension, that depends on the water model, enters Eq. 1, and hence the Rayleigh limit. Since the stability of a droplet depends on the chargesquared to surface tension ratio (considering volume constant), the surface tension for the specific water model will regulate the amount 
Table 1: Systems studied, radii and charge of the droplets. $n_{\mathrm{H}_{2} \mathrm{O}}$ denotes number of water molecules in the droplet and $n_{\mathrm{I}}$ denotes the number of ions in the aqueous droplet in the production run. $R_{e}[\mathrm{~nm}]$ is the radius of the equimolecular dividing surface (computed by using density of the TIP3P model at $350 \mathrm{~K}$ to be $0.9539 \mathrm{~g} / \mathrm{cm}^{3}$ and that of the TIP4P/2005 model $^{28}$ at $350 \mathrm{~K}$ to be $0.9713 \mathrm{~g} / \mathrm{cm}^{3}$. Q $Q_{\text {est }}$ (in units of elementary charge $e$ ) is the charge estimated at the Rayleigh limit, considering radius $R_{e}$. $R_{r}$ is the Rayleigh radius estimated considering the total droplet charge in the simulations. $R_{\text {sim }}[\mathrm{nm}]$ is the distance found from the concentration profiles in Fig. 4 at which the density becomes $5 \times 10^{-4} \mathrm{~g} / \mathrm{cm}^{3} . R_{\max }[\mathrm{nm}]$ is the distance from the droplet COM to the maximum of the ion concentration profile. $X$ is the fissility parameter, calculated by taking the square of the ratio between the charge of the droplet and $Q_{\text {est }}$. In all the systems the temperature is set at $T=350 \mathbf{K}$, besides those marked with an asterisk, which are found at $T=300 \mathrm{~K}$. The simulations were performed using the TIP3P water model, besides those marked with a dagger, where the TIP4P/2005 model was used.

\begin{tabular}{ccccccccc}
\hline System & $n_{\mathrm{H}_{2} \mathrm{O}}$ & $n_{\mathrm{I}}$ & $R_{e}$ & $Q_{\text {est }}$ & $R_{r}$ & $R_{\text {sim }}$ & $R_{\max }$ & $X$ \\
\hline 1 & 880 & $6 \mathrm{Na}^{+}$ & 1.88 & 7.9 & 1.56 & 2.6 & $0.90-0.95$ & 0.57 \\
2 & 5880 & $19 \mathrm{Na}^{+}$ & 3.65 & 20.6 & 3.37 & 4.4 & $2.65-2.70$ & 0.85 \\
3 & $2 \times 10^{4}$ & $36 \mathrm{Na}^{+}$ & 5.31 & 37.6 & 5.16 & 6.1 & $4.30-4.35$ & 0.92 \\
4 & $3 \times 10^{4}$ & $44 \mathrm{Na}^{+}$ & 6.08 & 46.0 & 5.90 & 6.8 & $5.10-5.15$ & 0.91 \\
5 & $3 \times 10^{4}$ & $44 \mathrm{Na}^{+}+$ & 6.08 & 46.0 & 5.90 & 6.8 & $5.10-5.15$ & 0.91 \\
& & $23 \mathrm{NaCl}^{2}$ & & & & & & \\
6 & $3 \times 10^{4}$ & $44 \mathrm{Cl}^{-}$ & 6.08 & 46.0 & 5.90 & 6.8 & $5.05-5.10$ & 0.91 \\
7 & $3 \times 10^{4}$ & $44 \mathrm{Cl}^{-}+$ & 6.08 & 46.0 & 5.90 & 6.8 & $5.10-5.15$ & 0.91 \\
& $23 \mathrm{NaCl}^{2}$ & & & & & & & \\
8 & $6 \times 10^{4}$ & $63 \mathrm{Na}^{+}$ & 7.66 & 65.1 & 7.50 & 8.3 & $6.90-6.95$ & 0.94 \\
\hline \hline 9 & 880 & $6 \mathrm{H}_{3} \mathrm{O}^{+}$ & 1.88 & 7.9 & 1.56 & 2.6 & $1.60-1.65$ & 0.57 \\
10 & $980^{*}$ & $8 \mathrm{H}_{3} \mathrm{O}^{+}$ & 1.92 & 8.2 & 1.92 & 2.6 & $1.80-1.85$ & 0.95 \\
\hline \hline 11 & 880 & $6 \mathrm{Cl}^{-}$ & 1.88 & 7.9 & 1.56 & 2.6 & $1.05-1.10$ & 0.57 \\
12 & $980^{*}$ & $8 \mathrm{Cl}^{-}$ & 1.92 & 8.2 & 1.92 & 2.6 & $1.15-1.20$ & 0.95 \\
\hline 13 & $3500^{\dagger}$ & $15 \mathrm{I}^{-}$ & 2.95 & 18.4 & 3.04 & 3.5 & $2.45-2.50$ & 0.65 \\
14 & $3500^{\dagger}$ & $16 \mathrm{Cl}^{-}$ & 2.95 & 18.4 & 3.04 & 3.5 & $2.20-2.25$ & 0.75 \\
15 & $3500^{\dagger}$ & $8 \mathrm{Cl}^{-}+$ & 2.95 & 18.4 & 3.04 & 3.5 & $2.15-2.20$ & 0.65 \\
& & $8 \mathrm{I}^{-}$ & & & & & $2.55-2.60$ &
\end{tabular}


of charge a droplet can support. Thus, fundamental questions, which include the validity of the Rayleigh limit droplets, and the factors that determine the ion spatial distribution should not be affected by the surface tension of the specific water model. Another important parameter that enters the ion distribution is the system's dielectric constant. On the one hand, the dielectric constant that enters Eq. 1 is that of the medium (vapor in our studies) surrounding the droplet because the Rayleigh model deals with a conducting droplet. On the other hand, the dielectric constant of the droplet determines the solvation energy of the ions in the Born's solvation model and the Debye length. ${ }^{34}$ Thus, the ion distribution may be affected by the dielectric constant of the water model. We expect that the majority of the ions will be found in the outer water shells of the droplets, thus they are subject to shape fluctuations and large density gradients. The shape fluctuations are coupled with the ion distribution. The distinct environment of the surface and the presence of ions will reduce the value of the dielectric constant considerably. ${ }^{35}$ Any water model will yield a lower dielectric constant on the droplet surface, thus it will be able to provide the trends in the spatial distribution of ions with the droplet size and the effect of counterions in the outer droplet layers. TIP3P predicts a closer value to the experimental dielectric constant than the TIP4P/2005.

\section{Test of a model hydronium force field}

The large number of studies on the Eigen ${ }^{36}$ and Zundel ${ }^{37}$ complexes of water with hydronium ion in small clusters are indicative of the challenges in modeling and experiments. ${ }^{38-57} \mathrm{Be}-$ cause of the large droplets that we study, we model the hydronium ions by a simple empirical model, where the parameters were taken from. ${ }^{58}$ We address the following questions: (i) Is there enough space on the droplet surface to accommodate the Eigen "pyramids". (ii) Does the particular structure of the Eigen complex, differentiate its location in a droplet relative to that of $\mathrm{Na}^{+}$ions?

To test the Eigen structure of the model we used, ${ }^{58}$ we carried out classical MD simulations on the protonated water cluster $\left(\mathrm{H}_{2} \mathrm{O}\right)_{30} \mathrm{H}_{3} \mathrm{O}^{+}$. The structures were compare with those by Ufimtsev et. al ${ }^{59}$ generated by ab initio MD (AIMD) perfromed at $T=300 \mathrm{~K}$. As there are multiple local energy minima (at finite $T$ ), a conventional simulation of the canonical ensemble (NVT) will not capture the structural isomers. Instead, simulated annealing were used to explore the complex energy landscape more reliably.

The simulation protocol is as follows: The system is heated by setting the temperature of the Langevin thermostat to $200 \mathrm{~K}$ for $0.5 \mathrm{~ns}$, then it is quenched by setting the temperature of the thermostat to $100 \mathrm{~K}$ for $0.5 \mathrm{~ns}$. The heating-cooling cycle is repeated 20 times and we observed distinct configurations between the cycles. We discard the trajectory where the temperature is $200 \mathrm{~K}$ and only use the $100 \mathrm{~K}$ trajectory for analysis.

At $T=100 \mathrm{~K}$, the hydronium ion forms an Eigen complex and reside on the surface of the cluster. We compare the hydroniumwater $\mathrm{O}-\mathrm{O}$ radial distribution function (RDF) shown in Fig. 2 with that of Ufimtsev et. al. ${ }^{59}$ The model hydronium model reproduces well the oxygen-oxygen distance of an Eigen complex $(2.6 \AA)$. In the RDF we observe a small shoulder centered at $2.9 \AA$ that is absent in the AIMD. The shoulder correspond to an oxygen that is 'colinear' with the hydrogen at the base of the $\mathrm{H}_{9} \mathrm{O}_{4}{ }^{+}$pyramid. The wide band from $\sim 4-4.8 \AA$ in our simulation is slightly shifted outwards compared to the AIMD simulation.

\section{Results and Discussion}

\section{Spatial distribution of $\mathrm{Na}^{+}$ions in aqueous droplets}

Fig. 3 (a) shows the oxygen-TIP3P density profile (O-DP) and the $\mathrm{Na}^{+}$concentration profile (CP) for two droplet sizes comprised (a) $2 \times 10^{4}$ TIP3P $\mathrm{H}_{2} \mathrm{O}-36 \mathrm{Na}^{+}$ions and (b) $3 \times 10^{4}$ TIP3P $\mathrm{H}_{2} \mathrm{O}-44 \mathrm{Na}^{+}$ions. The profiles have been nor- 


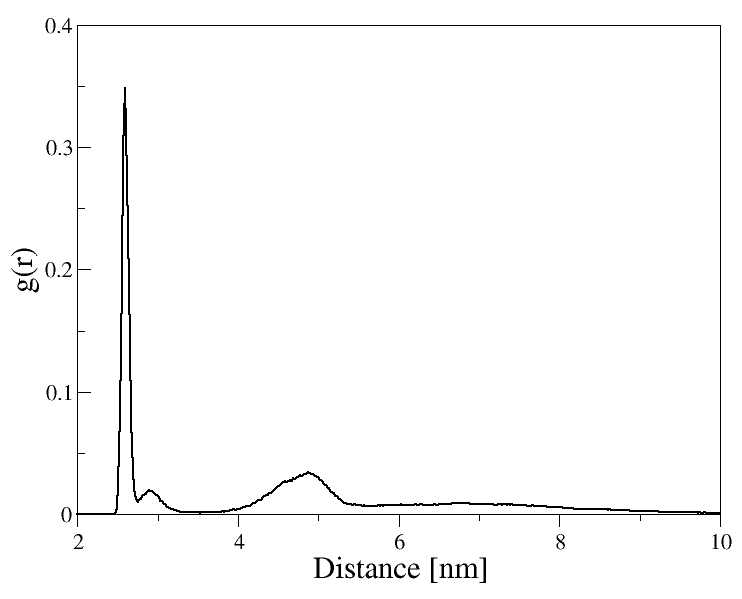

Figure 2: The $\mathrm{O}-\mathrm{O}$ radial distribution function between the hydronium ion and water molecules.

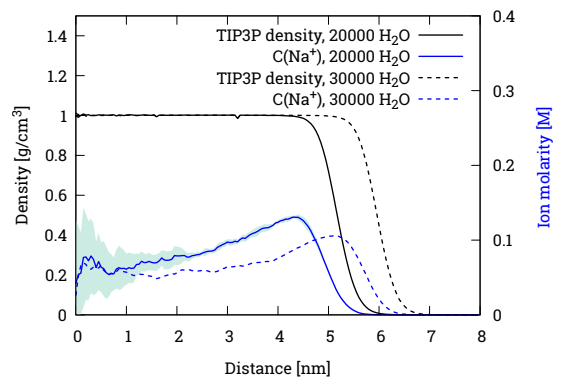

(a)

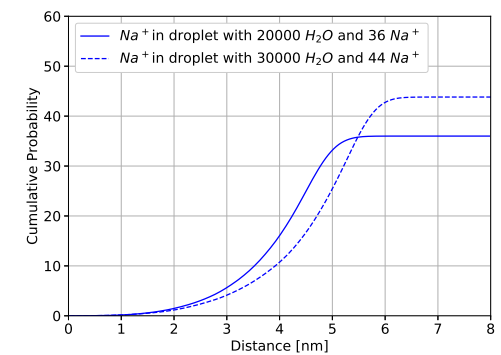

(b)

Figure 3: (a) Water density profiles (blue lines) and $\mathrm{Na}^{+}$concentration profiles (red lines) vs distance from the droplet $\mathrm{COM}$ for droplets comprised $2 \times 10^{4}$ TIP3P $\mathrm{H}_{2} \mathrm{O}-36 \mathrm{Na}^{+}$ions and $3 \times 10^{4}$ TIP3P $\mathrm{H}_{2} \mathrm{O}-44 \mathrm{Na}^{+}$ions at $T=350 \mathrm{~K}$. The blurry region shows the error bars. The standard deviation is calculated by using 5 blocks of raw data, where every block has $4 \times 10^{4}$ configurations. (b) Cumulative probability of the systems in (a).

malized by dividing with the volume element of a spherical shell $\left(\frac{4}{3} \pi\left[(r+d r)^{3}-r^{3}\right]\right.$ where $r$

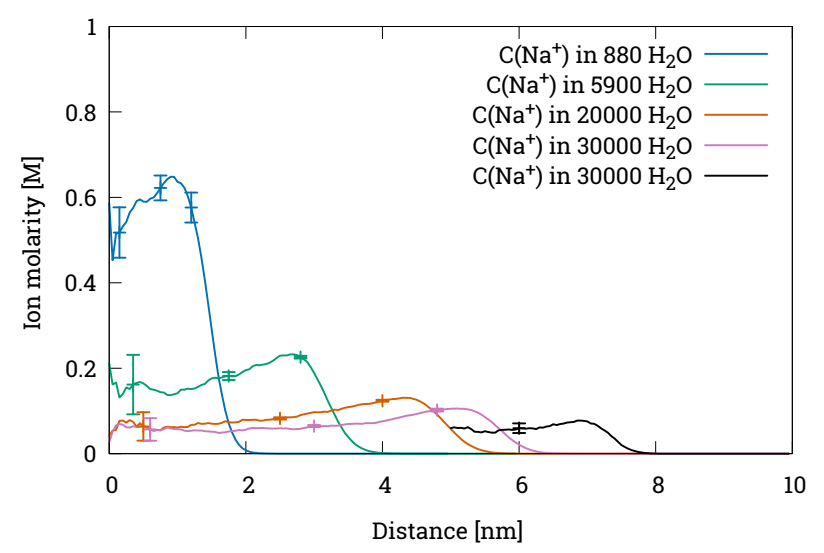

(a)

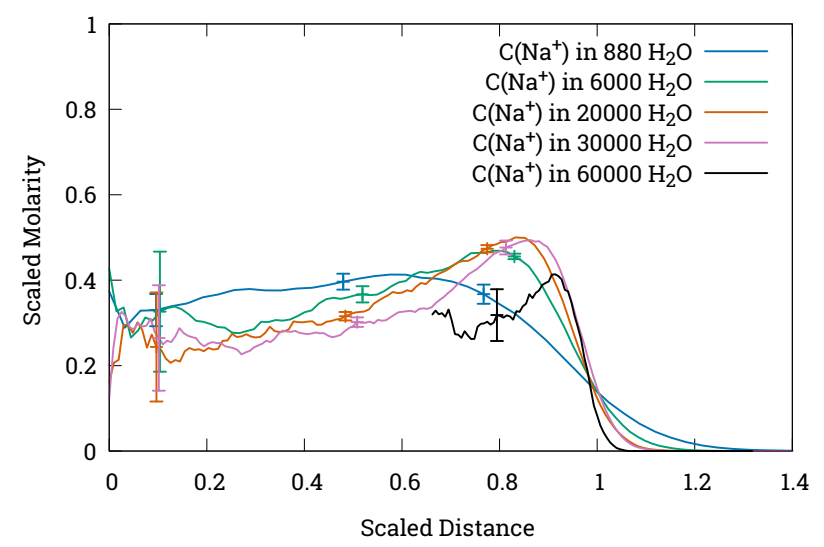

(b)

Figure 4: (a) $\mathrm{Na}^{+}$concentration profiles vs distance from the droplet COM for aqueous droplets of various sizes at $T=350 \mathrm{~K}$. Error bars are sparsely shown for clarity purpose. The error bars are shown in Fig. 3 and by Kwan et al. ${ }^{19}$ for droplets of 880 and 6000 water molecules. (b) Same as (a) but for scaled data. The $\mathrm{x}$-axis is scaled by the Rayleigh radius for the specific charge state of the droplet and the y-axis by $Q / R_{r}^{3}$ where $R_{r}$ is the Rayleigh radius and $Q$ the droplet charge (see data in Table 1 ).

is the droplet radius). The $\mathrm{Na}^{+}{ }_{-} \mathrm{CP}$ shows a broad maximum at $\approx 4.3 \mathrm{~nm}$ and $\approx 5.3 \mathrm{~nm}$ in the droplets of $2 \times 10^{4}$ and $3 \times 10^{4}$ TIP3P $\mathrm{H}_{2} \mathrm{O}$ molecule, respectively. In droplets sizes that we examined, the highest concentration of $\mathrm{Na}^{+}$ions is found in the outer $\mathrm{H}_{2} \mathrm{O}$ layers, where the density of $\mathrm{H}_{2} \mathrm{O}$ is still high (but still slightly lower than that at the droplet center). As shown in Table 1 the density in droplet sizes of a few tens of thousands of molecules diesoff to $5 \times 10^{-4} \mathrm{~g} / \mathrm{cm}^{3}$ at approximately $1.7 \mathrm{~nm}$ 
from the $\mathrm{Na}^{+}$concentration maximum. In the droplet comprised $6 \times 10^{4} \mathrm{H}_{2} \mathrm{O}$ molecules the distance is shorter, $1.4 \mathrm{~nm}$. In this $1.7 \mathrm{~nm}$ layer the water density decreases rapidly. Considering that the diameter of a TIP3P $\mathrm{H}_{2} \mathrm{O}$ molecule is $3.15 \AA$ and the radius of a $\mathrm{Na}^{+}$is $1.4 \AA$, the $1.7 \mathrm{~nm}$ layer would correspond approximately to five aqueous shells. The outer three shells are characterized by roughness and short dangling water chains.

Fig. 3 (b) shows the cumulative probability profiles (CPP) that result from the integration of $\mathrm{Na}^{+}-\mathrm{CP}$ (Fig. 3 (a)). The CPPs show three regions of different increase rates: (i) near the droplet COM the slope of the profiles is close to zero, (ii) in an intermediate distance from the droplet COM there is a rapid growth and (iii) near the droplet surface there is slower growth that leads to a plateau of the CPP. Specifically, for the droplet of $3 \times 10^{4}$ TIP3P $\mathrm{H}_{2} \mathrm{O}$ we find $\approx 15 \mathrm{Na}^{+}$ions in a spherical volume with radius $4.1 \mathrm{~nm}$ from the droplet COM. For radius greater than $4.1 \mathrm{~nm}$ the CPP increases rapidly up to $\approx 5.6 \mathrm{~nm}$ and that is followed by a slower saturation that reaches the value of $44 \mathrm{Na}^{+}$ions at $6.5 \mathrm{~nm}$. The profile of the droplet of $2 \times 10^{4}$, 880 and 6000 TIP3P $\mathrm{H}_{2} \mathrm{O}$ found in the SI (Fig. S1 (a), (b), (c)) show the same trend as that of the $3 \times 10^{4}$ TIP $3 P$ droplet. In the $2 \times 10^{4}$ TIP3P droplet, the majority of the ions are found in a radius of $3.8 \mathrm{~nm}-4.8 \mathrm{~nm}$ (1.0 nm thickness). In the $3 \times 10^{4}$ TIP3P the majority of the ions are in the spherical shell with radius $4.5 \mathrm{~nm}-5.8 \mathrm{~nm}$ (1.3 nm thickness). Overall, we find that in the sizes of droplets we have studied $\approx 23 \%-38 \%$ of the $\mathrm{Na}^{+}$ions are found in the region (i) of the CPP.

Figure 4 (a) shows the ion distributions for five droplet sizes. The largest droplet comprises $6 \times 10^{4}$ TIP3P $\mathrm{H}_{2} \mathrm{O}$ molecules and $63 \mathrm{Na}^{+}$ions. For the largest droplet we only show the distribution between $6.0 \mathrm{~nm}-8.0 \mathrm{~nm}$ where the statistics is reasonably good. The size of the droplet makes sufficient equilibration very challenging. Nevertheless, the region of the higher ion concentration emerges. Figure 4 (b) shows the scaled distributions, where the distance (x axis) in Fig. 4 (a) has been scaled by the Rayleigh radius shown in Table 1 and the y-axis by $Q / R_{r}^{3}$, where $R_{r}$ is the Rayleigh radius for the specific droplet charge and $Q$ is the droplet charge (radii and charges are presented in Table 1 ). The scaled ion distributions reveal an interesting behavior. The most striking feature is that the smaller the droplet the larger its extension in the scaled coordinates. We attribute this behavior in the pronounced shape fluctuations due its higher charge density relative to that of the other droplet sizes. We note here that the smallest droplet is at $X=0.57$ while the larger droplets are found at $X=0.85-0.94$. Since the shape fluctuations at $X=0.57$ are more pronounced relative to the larger droplets it is expected that even larger fluctuations at $X \approx 0.9$ will lead to an early droplet fragmentation. Even though the ion distribution profiles do not have any dynamic information, the size of the shape fluctuations for the smaller droplets predicts an early droplet fission. The early droplet fission does not imply that the Rayleigh limit does not hold. The early droplet fission is affected by the temperature. As can be seen in Table 1, a droplet of the same size at $300 \mathrm{~K}$ is found very close to the Rayleigh limit in simulations of several tens of nanoseconds. Another interesting feature is the shape of the ion distribution in the droplet of $880 \mathrm{H}_{2} \mathrm{O}$ molecules. The shape of the distribution is a broader peak than that of the ion distributions for the larger droplets. In order to test whether this broader distribution is the low value of $X$, simulations were performed for a droplet comprised $5990 \mathrm{H}_{2} \mathrm{O}$ molecules at $X=0.46$ (14 $\mathrm{Na}^{+}$ions) (Fig. S2 in SI). We found that the $\mathrm{Na}^{+}$distribution at lower $X$ does not become broader. Thus, we excluded the low value of $X$ as the factor that leads to a broader distribution.

We can recast Eq. 1 in the following form

$$
\frac{1}{\left(4 \pi \varepsilon_{0}\right)^{2}} \frac{Q^{2}}{R^{4}} R=|E|^{2} R=4 \varepsilon_{0} \gamma
$$

where $|E|$ is magnitude of the electric field of a spherical conductor. Equation 2 shows that the surface charge density (which is proportional to $|E|)^{60}$ is proportional to $1 / R^{1 / 2}$. The simulation data agree with the theoretical prediction 
(Eq. 2) that the surface charge density (which is proportional to ion concentration) decreases with the increase of the droplet radius as shown in Fig. 4 (a).

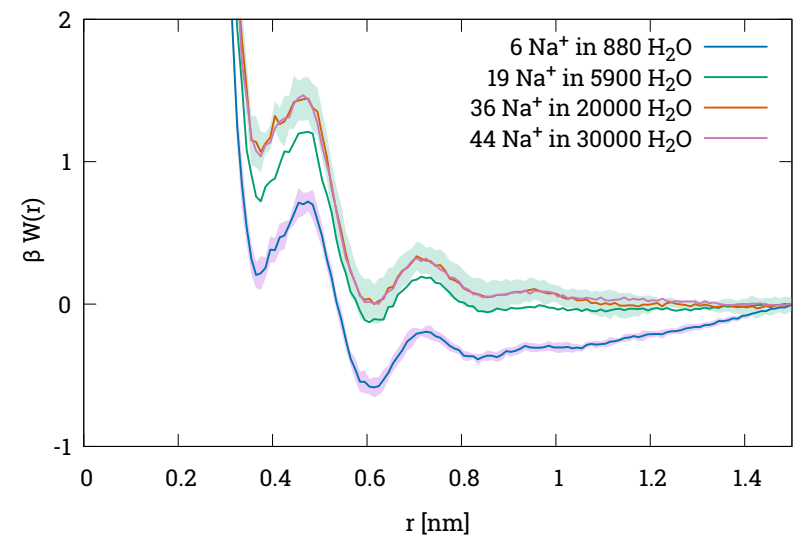

Figure 5: Potential of mean force (PMF) as a function of the distance between any pair of $\mathrm{Na}^{+}$ions in droplets of various sizes. The blurry regions show the error bars.

We computed the radial distribution function $(g(r))$ between the $\mathrm{Na}^{+}$ions (Fig. S3 in SI). We computed the potential of mean force (PMF) shown in Fig. 5 directly from the $g(r)$. The Na$\mathrm{Na}$ radial distribution function shows two significant peaks, at $\sim 3.7 \AA$ and $\sim 6.1 \AA$. The peaks correspond to contact ion pairs (CIP) and solvent separated ion pair (SSIP) respectively. A snapshot of each ion pairs are shown in Fig. S3 in SI. The location and ratio of the two peaks corresponding to the CIP and the SSIP agrees with earlier result obtained from bulk simulation of $0.3 \mathrm{M} \mathrm{NaCl}$ in TIP3P water. ${ }^{61}$

As the size of the droplet shrinks, the ion concentration needed to maintain the total charge of the droplet close to the Rayleigh limit increases. Savelyev et al. suggested that "in more dilute solutions the repulsion between like charged ions is stronger because they are less screened electrostatically by ions of the opposite charge", however the same trend holds in highly charged droplets without the presence of counterions. We suggest that as the ion concentration increases, the droplet become crowded and sodium ions are being 'forced' into solvent separated ion pairs.

\section{Effect of counterions}

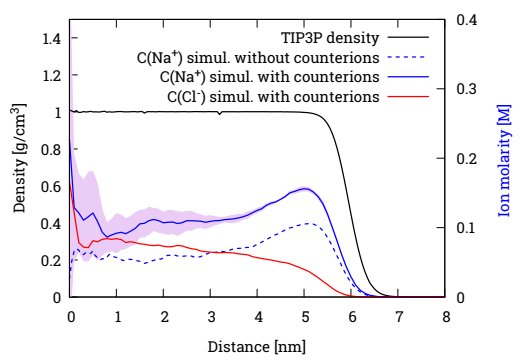

(a)

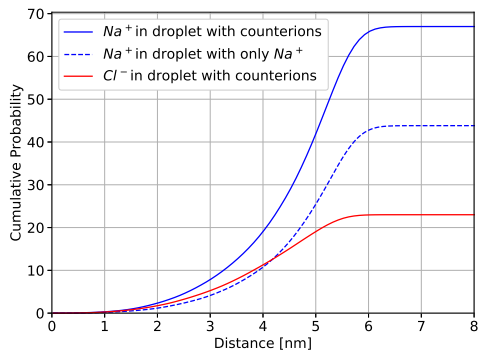

(b)

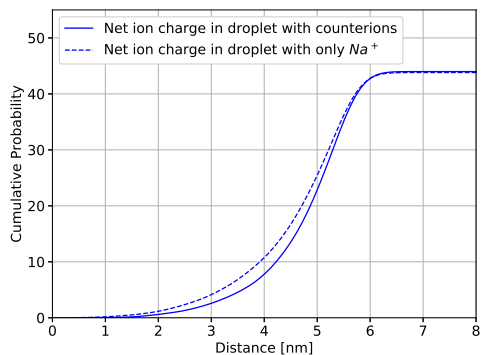

(c)

Figure 6: (a) Same as Fig. 3 (a) but for a droplet comprised $\approx 3 \times 10^{4} \mathrm{H}_{2} \mathrm{O}$ molecules, 44 $\mathrm{Na}^{+}$ions and $23 \mathrm{NaCl}$ pairs at $T=350 \mathrm{~K}$. The blurry region shows the error bars. The standard deviation is calculated by using 5 blocks of raw data, where every block has $4 \times 10^{4}$ configurations, separated by 0.2 ps. (b) Same as Fig. 3 (b) but for the droplet in (a). (c) $\mathrm{Cu}-$ mulative probability profiles of the net charge in droplets comprised $\approx 3 \times 10^{4} \mathrm{H}_{2} \mathrm{O}$ molecules - $44 \mathrm{Na}^{+}$only and the same droplet with additional $23 \mathrm{NaCl}$ pairs.

Figure 6 shows the spatial ion distribution of a droplet comprised $\approx 3 \times 10^{4} \mathrm{H}_{2} \mathrm{O}$ molecules, $44 \mathrm{Na}^{+}$ions and $23 \mathrm{NaCl}$ pairs. The distributions in the same size droplet but for excess $\mathrm{Cl}^{-}$ions are shown in Fig. S4 in the SI. A main feature in Fig. 6 (a) is that in a droplet with counterions the excess ions, $\mathrm{Na}^{+}$form an outer 
shell of higher concentration. The $\mathrm{Cl}^{-}$ions are depleted from the region where the $\mathrm{Na}^{+}$concentration is the highest. The $\mathrm{Cl}^{-}$concentration gradually increases toward the droplet center, where the $\mathrm{Na}^{+}$concentration is the lowest. In Figs S11, S12 and S13 in SI is shown that in aqueous droplets with $\mathrm{Cl}^{-}$only the $\mathrm{Cl}^{-}$show a clear propensity to be found near the surface. The fact that the $\mathrm{Cl}^{-}$are restricted in the droplet interior in the presence of the excess $\mathrm{Na}^{+}$is the result of the conducting character of the droplet, which dictates that the excess charge accumulates on the surface.

If we scale the $\mathrm{Na}^{+}$concentration in the presence of $\mathrm{Cl}^{-}$ions the two concentrations with and without counterions coincide. In the presence of counterions, the $\mathrm{Na}^{+}$concentration (not scaled) appear to extend more toward the outer $\mathrm{H}_{2} \mathrm{O}$ layers and to have higher concentration in the interior. This behavior is attributed to the fact that in the presence of counterions the concentration of $\mathrm{Na}^{+}$is higher in order to keep the overall charge of the droplet the same as without counterions.

Figure 6 (b) shows that the cumulative probability saturates at $6.2 \AA$ for $\mathrm{Na}^{+}$ions in droplet with and without $\mathrm{Cl}^{-}$counterions. The saturation point has been reached earlier for the $\mathrm{Cl}^{-}$ ions than that of the $\mathrm{Na}^{+}$ions. Figure $6(\mathrm{~b})$ shows that the net charge in the interior, estimated by subtracting the number of $\mathrm{Cl}^{-}$ions from that of $\mathrm{Na}^{+}$ions is lower in the droplets with counterions. This finding shows, that there is a higher concentration of $\mathrm{Na}^{+}$ions on the surface due to the presence of $\mathrm{NaCl}$. The increased concentration of $\mathrm{Na}^{+}$ion in the surface indicates that the surface is not saturated with charge, and it can accommodate a higher amount.

\section{Solvent structure induced by the $\mathrm{Na}^{+}$ions}

As expected the presence of the ions affect the solvent structure relative to that of the droplets without ions. In order to study the orientation of the water electric dipoles within the droplet, we compute $\cos (\phi)$, where $\phi$ is the angle between the $\mathrm{H}_{2} \mathrm{O}$ dipole moment (which is found

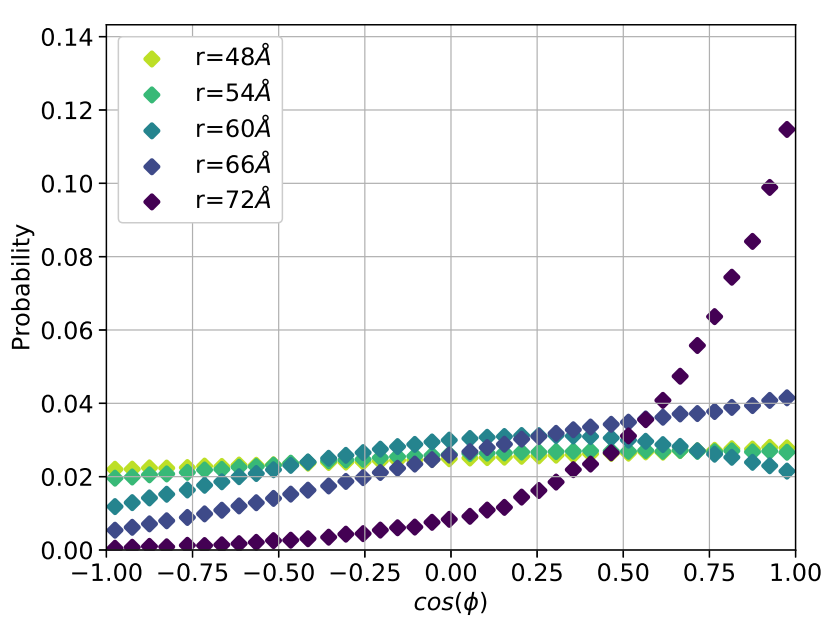

Figure 7: $\cos (\phi)$ distribution of the water electric dipole orientation in representative spherical shells of radius $r$ (shown in the legend) in a droplet comprised $\approx 3 \times 10^{4} \mathrm{H}_{2} \mathrm{O}$ molecules and 44 excess $\mathrm{Na}^{+}$ions. $\phi$ in defined in the text. The thickness of the spherical shell is 0.5 $\AA$.

along the $\mathrm{HOH}$ bisector, and starts at the oxygen site) and the vector that starts from the droplet center of mass (COM) and points to the oxygen site of a water molecule. Figure 7 shows the $\cos (\phi)$ distribution at representative spherical shells with center at the droplet COM. We focus in the region where the $\mathrm{Na}^{+}-\mathrm{CP}$ shows its maximum (48 $\AA-54 \AA)$ and beyond $(r>54 \AA)$. We find that in the outer water shells there is an overall shift toward the positive $\cos (\phi)$ values. At $48 \AA<r<54 \AA$ (which is the range of the maximum $\mathrm{Na}^{+}-\mathrm{CP}$ ) the range $-0.25<$ $\cos (\phi)<1.0$ is preferred. The $\cos (\phi)$ distribution in the $r$ values of the maximum of the $\mathrm{Na}^{+}-$ $\mathrm{CP}$ is to be contrasted with that of droplets of 1000 and $6000 \mathrm{H}_{2} \mathrm{O}$ discussed by Kwan et al. ${ }^{19}$ In the smaller droplets the preference for positive $\cos (\phi)$ values is more pronounced. This difference in the cosine distribution is attributed to the fact that the larger the droplet the lower the surface charge density. At $r>54 \AA$ the preference for the positive $\cos (\phi)$ becomes more pronounced. At the very outer water shells $(r \approx 72 \AA)$ the water molecules often form short dangling chains. The dangling water molecules are strongly oriented by the overall charge of the droplet. The distribution of $\cos (\phi)$ and of 
the orientation of the water molecule planes for $2 \times 10^{4}$ water molecules $-36 \mathrm{Na}^{+}$ions is shown in Fig. S8 in the SI. Similar orientation of the $\mathrm{H}_{2} \mathrm{O}$ dipoles in droplets of $3 \times 10^{4}-44 \mathrm{Na}^{+}$ ions to that of $2 \times 10^{4}-36 \mathrm{Na}^{+}$ions are also observed.

The comparison of the orientation of the water dipoles in the systems that we have studied (Table 1) revealed certain common features: (a) Within the vicinity of the maximal value of the $\mathrm{Na}^{+}-\mathrm{CP}$, the distribution of the $\cos (\phi)$ values increases almost linearly, with preference in the positive $\cos \phi$ values. The smaller the droplet, the larger the probability towards the $\cos (\phi)=$ 1.0 end. For a droplet of $\approx 880 \mathrm{H}_{2} \mathrm{O}-6 \mathrm{Na}^{+}$ions, the preferred orientation is for $0.25<\cos (\phi)<$ 1.0 , for a droplet of $\approx 6000 \mathrm{H}_{2} \mathrm{O}-6 \mathrm{Na}^{+}$is for $0.5<\cos (\phi)<1.0$ and for droplets of a few tens of thousand of molecules the distribution becomes more uniform but with a small preference for $\cos (\phi)>0.0$. (b) In the following water spherical shells of thickness of $\approx 4.5 \AA$ (1.5 $\mathrm{H}_{2} \mathrm{O}$ diameters), the distribution of $\cos (\phi)$ is an inverted parabola with maximum at approximately $\cos (\phi)=0.25$. The maximum is not representative of the majority of the dipole orientations. We have to consider the vicinity of the maximum. The dominant part of the $\cos (\phi)$ distribution spreads from the positive range up to the negative range. For the $\approx 6000 \mathrm{H}_{2} \mathrm{O}-6 \mathrm{Na}^{+}$the dominant part is in the range of $-0.25<\cos \theta<0.75$ and for droplets of a few tens of thousand of molecules is $-0.5<\cos \phi<1$ The larger the droplet, the less pronounced the parabola is, and a larger range of $\cos (\phi)$ values is preferred. (c) In the very outer shell, there is formation of short dangling $\mathrm{H}_{2} \mathrm{O}$ strings that show elastic motion. The $\mathrm{H}_{2} \mathrm{O}$ molecules is these strings are strongly oriented by the overall droplet electric field.

Figure S9 in the SI shows the distribution of $\cos (\phi)$ for the droplets of $3 \times 10^{4} \mathrm{H}_{2} \mathrm{O}-44$ $\mathrm{Na}^{+}$ions - $23 \mathrm{NaCl}$ pairs. The distributions of Fig. S9 and Fig. 7 are identical, which reinforces our argument that it is the overall charge of the droplet that determines the orientation of the $\mathrm{H}_{2} \mathrm{O}$ and not the specific ions.

In order to characterize further the orientation of the water molecules, we also compute

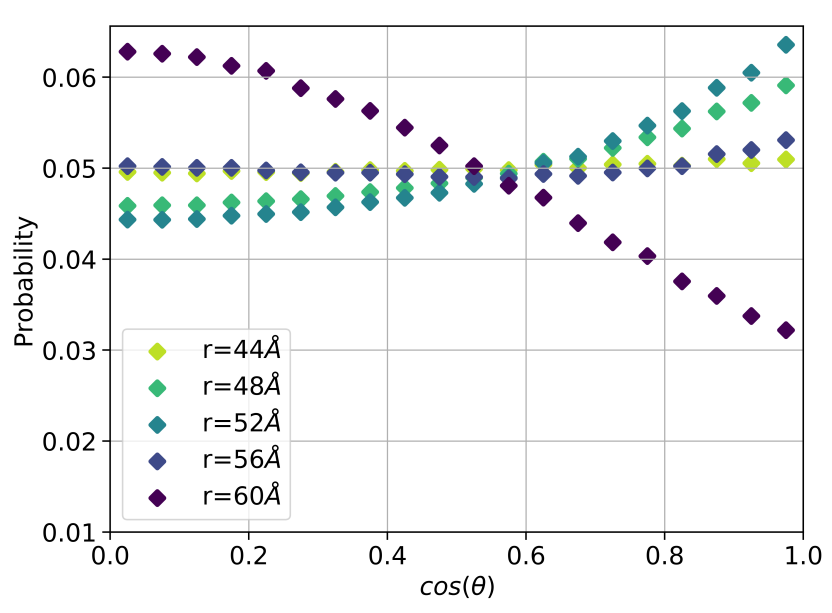

Figure 8: $\cos (\theta)$ distribution in representative spherical shells of radius $r$ (shown in the legend) in a droplet comprised $\approx 2 \times 10^{4} \mathrm{H}_{2} \mathrm{O}$ molecules and 36 excess $\mathrm{Na}^{+}$ions. $\theta$ in defined in the text. The thickness of the spherical shell is $0.5 \AA$.

the planar angle $\theta$, defined as the angle between the normal vector of the plane of the water molecule and the vector that starts from the droplet center of mass (COM) and points to the oxygen site of a water molecule. The possible range of $\cos (\theta)$ is dependent on $\cos (\phi)$, where $\cos ^{2}(\phi)+\cos ^{2}(\theta)<1$. In addition, since the sign of the normal vector is arbitrary and using the trigonometric identity:

$$
\cos (\theta)=\frac{\mathbf{a} \cdot \mathbf{b}}{|\mathbf{a}||\mathbf{b}|}=\cos (-\theta)=\frac{\mathbf{a} \cdot(-\mathbf{b})}{|\mathbf{a}||\mathbf{b}|}
$$

We can limit the range of $\cos (\theta)$ to $[0,1]$. However, it is possible that the shift to positive of the dipole angle $\theta$ leads to the shift of the plane angle to positive. For a droplet of $2 \times 10^{4} \mathrm{H}_{2} \mathrm{O}-36 \mathrm{Na}^{+}$ions the maximum of the ion distribution is approximately in the range of $4.0 \mathrm{~nm}<r<4.8 \mathrm{~nm}$. In the interior of a charged droplet (up to $46 \AA$ ), the distribution of $\cos (\theta)$ is uniform, which indicates that all the plane orientations are equally likely. In the next aqueous shells, up to approximate the Rayleigh radius $(5.2 \mathrm{~nm}-5.4 \mathrm{~nm})$ the $\cos (\theta)$ shows a propensity for $\cos (\theta)>0.5$, which indicates that the water plane is tilted on the spherical surface. Further away, the $\cos (\theta)$ distribution becomes uniform once more and in 
the dangling water $\cos (\theta)$ shows a propensity for values close to zero.

\section{Spatial distribution of hydronium ions}

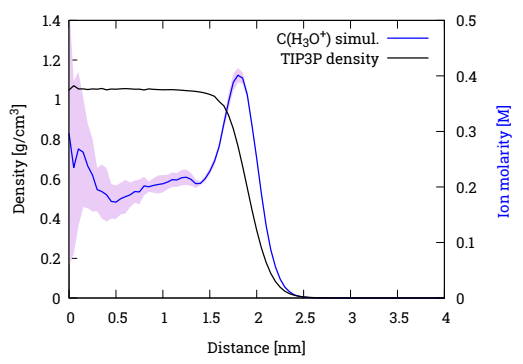

(a)

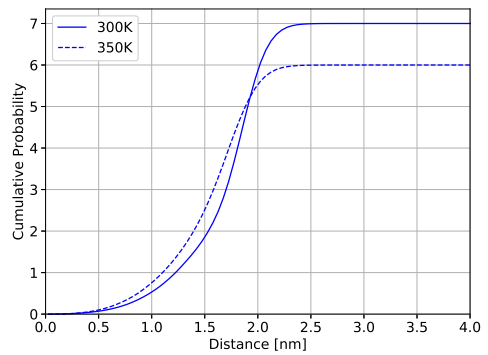

(b)

Figure 9: (a) Droplet density and hydronium ion concentrations. Equilibrated ion distribution in a drop comprised $\approx 1000 \mathrm{H}_{2} \mathrm{O}$ molecules and $8 \mathrm{H}_{3} \mathrm{O}^{+}$ions at $T=300 \mathrm{~K}$. The blurry region shows the error bars. The production run is for $40 \mathrm{~ns}$. The standard deviation was calculated by using 5 blocks of raw data, each of 40000 frames. (b) Cumulative probability function that arises from the integral of (a).

Figure 9 (a) shows the distribution of "hydronium" ions in a droplet of $\approx 1000 \mathrm{H}_{2} \mathrm{O}$ molecules and 8 ions. The general features of the distribution are the same as that of the $\mathrm{Na}^{+}$ ions but the hydronium reside much more in the outer water shells of the droplet than the $\mathrm{Na}^{+}$ ions. Even though the hydronium model omits important quantum effects it demonstrates that certain positively charged ions may found in the region of low water density on the surface. In general we expect that very small ions such as $\mathrm{Li}^{+}$and large ions will be found further out than $\mathrm{Na}^{+}$. Small ions like $\mathrm{Li}^{+}$are surrounded by a rigidly bound first solvation that makes them similar to a large ion like $\mathrm{Cs}^{+}$. The orientation of the water dipoles and of the planes are shown in Fig. 10.

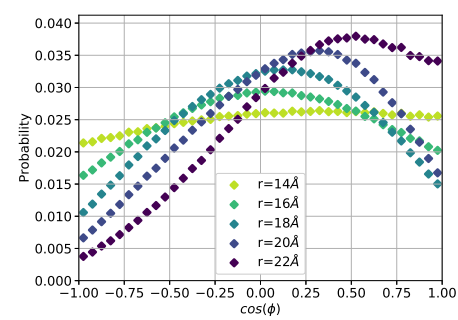

(a)

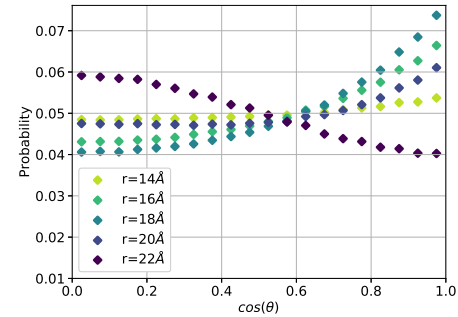

(b)

Figure 10: Dipole orientation and plane orientation in droplets comprised 1000 TIP3P water molecules and $8 \mathrm{H}_{3} \mathrm{O}^{+}$ions.

\section{Conclusion}

We have performed a systematic study of the distribution of positive and negative ions in a nano-drop with and without counterions. We conclude that: (a) For droplets that range in size between several hundreds to several tens of thousands of water molecules the maximum of the $\mathrm{Na}^{+}$spatial distribution is situated in the same depth from the droplet very outer surface. This depth is $\approx 1.7 \mathrm{~nm}$ from the point that the water density becomes $5 \times 10^{-4} \mathrm{~g} / \mathrm{cm}^{3}$. The difference between the $R_{e}$ and the maximum of the $\mathrm{Na}^{+}$distribution is approximately $1.0 \mathrm{~nm}$. We expect that for even microscopic droplets the location of the $\mathrm{Na}^{+}$ions will be maintained. (b) For liquid droplets of diameter $5 \mathrm{~nm}$ (which is $\approx 1000 \mathrm{H}_{2} \mathrm{O}$ molecules) to $14 \mathrm{~nm}$ (which is $\approx 3 \times 10^{5} \mathrm{H}_{2} \mathrm{O}$ molecules) the maximum of the $\mathrm{Na}^{+}$concentration is not significantly higher than that in the droplet interior. Especially, for the smallest droplet of $5 \mathrm{~nm}$ diameter, the ion distribution is very broad. (c) Scaling of the 
$\mathrm{x}$-axis of the ion distributions by the Rayleigh radius of the droplet, reveals that for droplets with diameter, $7 \mathrm{~nm}$ (which is $\approx 6 \times 10^{3} \mathrm{H}_{2} \mathrm{O}$ molecules) to $14 \mathrm{~nm}$ the ion distribution profiles almost coincide. For the droplet of diameter $5 \mathrm{~nm}\left(\approx 1000 \mathrm{H}_{2} \mathrm{O}\right.$ molecules $)$ the $\mathrm{Na}^{+}$ scaled concentration profile differs from the others. The profile indicates that the smallest droplet undergoes larger shape fluctuations relative to its dimensions. (d) The nature of the ions plays a very significant role in its location in droplet as indicate by comparing the distribution of the $\mathrm{Na}^{+}, \mathrm{Cl}^{-} \mathrm{I}^{-}$and that of a model hydronium ion. (e) The presence of counterions plays a dramatic role in the manner that the ions are distributed. The ions in excess are situated in the exterior vs the counterions (ions in deficiency). The arrangement of the ions is reminiscent of that of a spherical capacitor.

In our analysis we expressed the Rayleigh limit using the macroscopic expression. As we have shown in previous work, ${ }^{13,62}$ for droplets of a few hudrends of water molecules we can define the Rayleigh limit by finding when the free energy profile of the ion release reaches $\mathrm{kT}$. For systems that are that small, the number of water molecules and not the radius are possibly better variables for describing when the free energy barrier of the fragmentation process approaches zero.

There are still a number of open questions to address such as that of the effect of the size of the ions in their spatial distribution. Another question is the location of a macroion in a droplet in the presence of counterions. ${ }^{63} \mathrm{It}$ is expected that negative ions will condense on a positive charged macroion. A possible location of the macroion in the outer layers will affect its reactivity because of the different ionic strength on droplet outer layers vs the interior. This effect is expected to emerge in larger droplets where there is enough volume so as the macroion is solvated and there is a large number of counterions. We suggest that large droplets of at least $20 \mathrm{~nm}$ in diameter have to be simulated in order this effect to emerge.

\section{Supplementary Material}

See supplementary material of (a) Details of the force field parameters; (b) Comparison of $\mathrm{Na}^{+}$, $\mathrm{Cl}^{-}, \mathrm{I}^{-}$distribution profiles; $\mathrm{Na}^{+}-\mathrm{Na}^{+}$radial distribution profiles.

Acknowledgement S.C. greatly thanks Prof. D. Frenkel, Department of Chemistry, University of Cambridge, UK, Prof. S. S. Xantheas, Pacific Northwest National Laboratory and Dr. Anatoly Malevanets, University of Western Ontario for discussions on the stability of charged systems. S.C. acknowledges an NSERC-Discovery grant (Canada) for funding this research. Sci-Net, SHARCNET and Compute Canada are acknowledged for providing the computing facilities.

\section{References}

(1) Rezaee, M.; Assadi, Y.; Hosseini, M.R. M.; Aghaee, E.; Ahmadi, F.; Berijani, S. Determination of organic compounds in water using dispersive liquidliquid microextraction. J. Chromatogr. A 2006, 1116, 1-9.

(2) Bain, R. M.; Pulliam, C. J.; Cooks, R. G. Accelerated Hantzsch electrospray synthesis with temporal control of reaction intermediates. Chem. Sci. 2015, 6, 397-401.

(3) Chen, X.; Cooks, R. G. Accelerated reactions in field desorption mass spectrometry. J. Mass Spectrom. 2018, 53, 942-946.

(4) Schrader, R. L.; Fedick, P. W.; Mehari, T. F.; Cooks, R. G. Accelerated Chemical Synthesis: Three Ways of Performing the Katritzky Transamination Reaction. J. Chem. Educ. 2019,

(5) Lee, J. K.; Banerjee, S.; Nam, H. G.; Zare, R. N. Acceleration of reaction in charged microdroplets. Q. Rev. Biophys. 2015, 48, 437-444.

(6) Ingram, A. J.; Boeser, C. L.; Zare, R. N. Going beyond electrospray: mass spectro- 
metric studies of chemical reactions in and on liquids. Chem. Sci. 2016, 7, 39-55.

(7) Consta, S.; Kapral, R. Dynamics of proton transfer in mesoscopic clusters. J. Chem. Phys. 1996, 104, 4581-4590.

(8) Consta, S.; Kapral, R. Proton transfer in mesoscopic, molecular clusters. J. Chem. Phys. 1994, 101, 10908-10914.

(9) Wilm, M. Principles of electrospray ionization. Mol. Cell. Proteomics 2011, 10, M111-009407.

(10) Mather, T.; Harrison, R. Electrification of volcanic plumes. Surv. Geophys. 2006, 27, 387-432.

(11) Iribarne, J. V.; Thomson, B. A. On the evaporation of small ions from charged droplets. J. Chem. Phys. 1976, 64, 22872294.

(12) Thomson, B.; Iribarne, J. Field induced ion evaporation from liquid surfaces at atmospheric pressure. J. Chem. Phys. 1979, $71,4451-4463$.

(13) Consta, S.; Mainer, K. R.; Novak, W. Fragmentation mechanisms of aqueous clusters charged with ions. J. Chem. Phys. 2003, 119, 10125-10132.

(14) Rayleigh, L. XX. On the equilibrium of liquid conducting masses charged with electricity. Philos. Mag. 1882, 14, 184186.

(15) Hendricks, C.; Schneider, J. Stability of a conducting droplet under the influence of surface tension and electrostatic forces. Am. J. Phys 1963, 31, 450-453.

(16) Peters, J. Rayleigh's electrified water drops. Eur. J. Phys. 1980, 1, 143.

(17) Consta, S.; Malevanets, A. Disintegration mechanisms of charged nanodroplets: novel systems for applying methods of activated processes. Mol. Simul. 2015, 41, 73-85.
(18) Oh, M. I.; Malevanets, A.; Paliy, M.; Frenkel, D.; Consta, S. When droplets become stars: charged dielectric droplets beyond the Rayleigh limit. Soft Matter 2017, 13, 8781-8795.

(19) Kwan, V.; Malevanets, A.; Consta, S. Where Do the Ions Reside in a Highly Charged Droplet? J. Phys. Chem. A 0, 0, null, PMID: 31589448.

(20) Jungwirth, P.; Tobias, D. J. Specific ion effects at the air/water interface. Chem. Rev. 2006, 106, 1259-1281.

(21) Dang, L. X.; Schenter, G. K. Rate theory of ion pairing at the water liquid-vapor interface: A case of sodium iodide. J. Chem. Phys. 2018, 148, 222820.

(22) Nguyen, M.; Rick, S. W. The influence of polarizability and charge transfer on specific ion effects in the dynamics of aqueous salt solutions. J. Chem. Phys. 2018, 148, 222803.

(23) Smith, J.; Rick, S. W. Ion clustering in aqueous salt solutions near the liquid/vapor interface. arXiv preprint arXiv:1603.07106 2016,

(24) Petersen, P. B.; Saykally, R. J. On the nature of ions at the liquid water surface. Annu. Rev. Phys. Chem. 2006, 5\%, 333364.

(25) Phillips, J. C.; Braun, R.; Wang, W.; Gumbart, J.; Tajkhorshid, E.; Villa, E.; Chipot, C.; Skeel, R. D.; Kalé, L.; Schulten, K. Scalable molecular dynamics with NAMD. J. Comput. Chem. 2005, 26, 1781-1802.

(26) Humphrey, W.; Dalke, A.; Schulten, K. VMD: Visual Molecular Dynamics. $J$. Mol. Graphics 1996, 14, 33-38.

(27) Jorgensen, W. L.; Jenson, C. Temperature dependence of TIP3P, SPC, and TIP4P water from NPT Monte Carlo simulations: Seeking temperatures of maximum density. J. Comput. Chem. 1998, 19, 1179 1186. 
(28) Abascal, J. L. F.; Vega, C. A general purpose model for the condensed phases of water: TIP4P/2005. J. Chem. Phys. 2005, 123, 234505.

(29) Noskov, S. Y.; Roux, B. Control of Ion Selectivity in LeuT: Two Na+ Binding Sites with Two Different Mechanisms. J. Mol. Biol. 2008, 377, $804-818$.

(30) Beglov, D.; Roux, B. Finite representation of an infinite bulk system: Solvent boundary potential for computer simulations. $J$. Chem. Phys. 1994, 100, 9050-9063.

(31) Chandrasekhar, J.; Spellmeyer, D. C.; Jorgensen, W. L. Energy component analysis for dilute aqueous solutions of lithium(1+), sodium(1+), fluoride(1-), and chloride(1-) ions. J. Am. Chem. Soc. 1984, 106, 903-910.

(32) McDonald, N. A.; Duffy, E. M.; Jorgensen, W. L. Monte Carlo Investigations of Selective Anion Complexation by a Bis(phenylurea) p-tert-Butylcalix[4]arene. J. Am. Chem. Soc. 1998, 120, 5104-5111.

(33) Vega, C.; de Miguel, E. Surface tension of the most popular models of water by using the test-area simulation method. J. Chem. Phys. 2007, 126, 154707.

(34) Varela, L. M.; Garcıa, M.; Mosquera, V. Exact mean-field theory of ionic solutions: non-Debye screening. Phys. Rep. 2003, 382, 1-111.

(35) Elton, D. C.; Fernández-Serra, M.-V. Polar nanoregions in water: A study of the dielectric properties of TIP4P/2005, TIP4P/2005f and TTM3F. J. Chem. Phys. 2014, 140, 124504.

(36) Eigen, M. Proton transfer, acid-base catalysis, and enzymatic hydrolysis. Part I: elementary processes. Angew. Chem. Int. Ed. 1964, 3, 1-19.

(37) Schuster, P.; Zundel, G.; Sandorfy, C. Hydrogen bond; recent developments in theory and experiments; North-Holland Publishing Company, 1976.
(38) Asmis, K. R.; Pivonka, N. L.; Santambrogio, G.; Brümmer, M.; Kaposta, C.; Neumark, D. M.; Wöste, L. Gas-phase infrared spectrum of the protonated water dimer. Science 2003, 299, 1375-1377.

(39) Vendrell, O.; Gatti, F.; Meyer, H.-D. Dynamics and infrared spectroscopy of the protonated water dimer. Angew. Chem. Int. Ed. 2007, 46, 6918-6921.

(40) Valeev, E. F.; Schaefer III, H. F. The protonated water dimer: Brueckner methods remove the spurious $\mathrm{C} 1$ symmetry minimum. J. Chem. Phys. 1998, 108, 71977201.

(41) Vendrell, O.; Gatti, F.; Meyer, H.-D. Full dimensional (15-dimensional) quantumdynamical simulation of the protonated water dimer. II. Infrared spectrum and vibrational dynamics. J. Chem. Phys. 2007, 127, 184303.

(42) Pieniazek, P. A.; VandeVondele, J.; Jungwirth, P.; Krylov, A. I.; Bradforth, S. E. Electronic structure of the water dimer cation. J. Phys. Chem. A 2008, 112, 6159-6170.

(43) Spura, T.; Elgabarty, H.; Kühne, T. D. "On-the-fly" coupled cluster path-integral molecular dynamics: impact of nuclear quantum effects on the protonated water dimer. Phys. Chem. Chem. Phys. 2015, 17, 14355-14359.

(44) Auer, A. A.; Helgaker, T.; Klopper, W. Accurate molecular geometries of the protonated water dimer. Physical Chemistry Chemical Physics 2000, 2, 2235-2238.

(45) Wales, D. J. Rearrangements and tunneling splittings of protonated water dimer. J. Chem. Phys. 1999, 110, 10403-10409.

(46) Chang, H.-C.; Wu, C.-C.; Kuo, J.-L. Recent advances in understanding the structures of medium-sized protonated water clusters. Int. Rev. Phys. Chem. 2005, 24, 553-578. 
(47) Tachikawa, M.; Shiga, M. Geometrical $\mathrm{H} / \mathrm{D}$ isotope effect on hydrogen bonds in charged water clusters. J. Am. Chem. Soc. 2005, 127, 11908-11909.

(48) Park, M.; Shin, I.; Singh, N. J.; Kim, K. S. Eigen and Zundel forms of small protonated water clusters: structures and infrared spectra. J. Phys. Chem. A 2007, 111, 10692-10702.

(49) Wang, H.; Agmon, N. Reinvestigation of the infrared spectrum of the gas-phase protonated water tetramer. The Journal of Physical Chemistry A 2017, 121, 30563070 .

(50) Mouhat, F.; Sorella, S.; Vuilleumier, R.; Saitta, A. M.; Casula, M. Fully quantum description of the Zundel ion: combining variational quantum Monte Carlo with path integral Langevin dynamics. Journal of chemical theory and computation 2017, 13, 2400-2417.

(51) Xantheas, S. S.; Dunning Jr, T. H. Ab initio studies of cyclic water clusters $(\mathrm{H} 2 \mathrm{O})$ $\mathrm{n}, \mathrm{n}=1-6$. I. Optimal structures and vibrational spectra. J. Chem. Phys. 1993, 99, 8774-8792.

(52) Yu, Q.; Bowman, J. M. High-level quantum calculations of the IR spectra of the Eigen, Zundel, and ring isomers of $\mathrm{H}+$ (H2O) 4 find a single match to experiment. J. Am. Chem. Soc. 2017, 139, 1098410987.

(53) Yu, Q.; Bowman, J. M. Classical, Thermostatted Ring Polymer, and Quantum VSCF/VCI Calculations of IR Spectra of $\mathrm{H} 7 \mathrm{O} 3+$ and H9O4+ (Eigen) and Comparison With Experiment. J. Phys. Chem. A 2019,

(54) Burnham, C. J.; Petersen, M. K.; Day, T. J.; Iyengar, S. S.; Voth, G. A. The properties of ion-water clusters. II. Solvation structures of $\mathrm{Na}+, \mathrm{Cl}-$, and $\mathrm{H}+$ clusters as a function of temperature. $J$. Chem. Phys. 2006, 124, 024327.
(55) Petersen, M. K.; Iyengar, S. S.; Day, T. J.; Voth, G. A. The hydrated proton at the water liquid/vapor interface. J. Phys. Chem. B 2004, 108, 14804-14806.

(56) Hvelplund, P.; Kurten, T.; Støchkel, K.; Ryding, M. J.; Nielsen, S. B.; Uggerud, E. Stability and Structure of Protonated Clusters of Ammonia and Water, $\mathrm{H}+$ (NH3) $\mathrm{m}(\mathrm{H} 2 \mathrm{O})$ n. J. Phys. Chem. A 2010, 114, 7301-7310.

(57) Silverstein, T. P. The Aqueous Proton Is Hydrated by More Than One Water Molecule: Is the Hydronium Ion a Useful Conceit? J. Chem. Educ. 2014, 91, 608-610.

(58) Chuev, G. N.; Fedorov, M. V.; Chiodo, S.; Russo, N.; Sicilia, E. Hydration of ionic species studied by the reference interaction site model with a repulsive bridge correction. J. Comp. Chem. 2008, 29, 24062415 .

(59) Ufimtsev, I. S.; Martinez, T. J. Quantum chemistry on graphical processing units. 3. Analytical energy gradients, geometry optimization, and first principles molecular dynamics. J. Chem. Theory Comput. 2009, 5, 2619-2628.

(60) Jackson, J. D. Classical Electrodynamics, third ed. ed.; John Wiley \& Sons: New York, NY, 1998.

(61) Savelyev, A.; Papoian, G. A. Molecular Renormalization Group Coarse-Graining of Electrolyte Solutions: Application to Aqueous $\mathrm{NaCl}$ and $\mathrm{KCl}$. J. Phys. Chem. B 2009, 113, 7785-7793, PMID: 19425537.

(62) Consta, S. Fragmentation reactions of charged aqueous clusters. J. Mol. Struct. THEOCHEM 2002, 591, 131-140.

(63) Sharawy, M.; Consta, S. Effect of counterions on the charging mechanisms of a macromolecule in aqueous nanodrops. $J$. Chem. Phys. 2014, 141, 104321. 